\title{
On automorphism groups of quasiprimitive 2-arc transitive graphs
}

\author{
Cai Heng Li
}

Received: 26 November 2006 / Accepted: 5 September 2007 / Published online: 6 October 2007

(C) Springer Science+Business Media, LLC 2007

\begin{abstract}
We characterize the automorphism groups of quasiprimitive 2-arc-transitive graphs of twisted wreath product type. This is a partial solution for a problem of Praeger regarding quasiprimitive 2-arc transitive graphs. The solution stimulates several further research problems regarding automorphism groups of edge-transitive Cayley graphs and digraphs.
\end{abstract}

Keywords Quasiprimitive · 2-arc transitive · Automorphisms · Cayley graphs

\section{Introduction}

In a graph $\Gamma$, an ordered pair of adjacent vertices is called an $\operatorname{arc}$, and a sequence of distinct vertices $(u, v, w)$ is called a 2-arc if $v$ is adjacent to both $u$ and $w$. A graph $\Gamma$ is called $(X, 2)$-arc transitive, where $X \leq$ Aut $\Gamma$, if $X$ acts transitively on the set of 2 -arcs of $\Gamma$.

The class of 2-arc transitive graphs is one of the central objects in algebraic graph theory, which have been intensively studied in the literature, see for example [1, 7, $12,13,17]$ and references therein. In particular, Praeger [13] gave a reduction for the study of finite non-bipartite 2-arc transitive graphs, which leads to the study of quasiprimitive 2-arc transitive graphs, defined later. The main purpose of this paper is to characterize the automorphism group for one type of quasiprimitive 2-arc transitive graph.

This work forms part of an ARC grant project and is supported by a QEII Fellowship.

C.H. Li $(\bowtie)$

School of Mathematics and Statistics, The University of Western Australia, Crawley, WA 6009, Australia

e-mail: li@maths.uwa.edu.au 
Theorem 1.1 Let $\Gamma$ be a finite $(X, 2)$-arc-transitive graph. Assume that $X$ has a unique minimal normal subgroup $G$, which is non-Abelian and regular on the vertex set of $\Gamma$. Then $\Gamma$ is not 3-arc transitive, and either

(i) $\Gamma$ has valency 8 , and Aut $\Gamma=\left(G \times \mathbb{Z}_{2}^{3}\right) \rtimes \mathrm{GL}(3,2)$, or

(ii) $G$ is the unique minimal normal subgroup of Aut $\Gamma$.

A permutation group $G$ on a set $\Omega$ is said to be quasiprimitive if every non-trivial normal subgroup of $G$ is transitive. Praeger [13] showed that only four of the eight types of quasiprimitive permutation groups can occur as automorphism groups of 2-arc transitive graphs, which are described below. Let $X$ be a quasiprimitive permutation group on $\Omega$, and let $\operatorname{soc}(X)$ be the socle of $X$, that is the product of all minimal normal subgroups of $X$. The four types involved in the study of 2-arc transitive graphs are

HA Holomorph Affine type: $\operatorname{soc}(X)$ is elementary Abelian, regular on $\Omega$.

AS Almost Simple type: $\operatorname{soc}(X)$ is a non-Abelian simple group.

PA Product Action type: $\operatorname{soc}(X)$ is a non-Abelian non-simple minimal normal subgroup of $X$, and $\operatorname{soc}(X)$ has no normal subgroup which is regular on $\Omega$.

TW Twisted Wreath product type: $\operatorname{soc}(X)$ is a non-Abelian non-simple minimal normal subgroup of $X$, and $\operatorname{soc}(X)$ acts regularly on $\Omega$.

A graph $\Gamma$ is called quasiprimitive $(X, 2)$-arc transitive if $X$ is quasiprimitive on $V \Gamma$ and 2-arc transitive on $\Gamma$. A classification of quasiprimitive $(X, 2)$-arc transitive graphs of type HA is given in [7]; a description of those of type TW is given in [1]; examples of type PA are given in [12]. In the study of such graphs, the problem of determining their automorphism groups naturally occurs, refer to [15] and [5, 7, 8]. Theorem 1.1 determines the automorphism groups for quasiprimitive 2-arc transitive graphs of twisted wreath product type.

\section{Preliminary results}

In this section we collect notation and preliminary results which will be used in the ensuing sections. For a group $X$ and a subgroup $H<X$, denote by $\mathbf{N}_{X}(H)$ and $\mathbf{C}_{X}(H)$ the normalizer and the centralizer of $H$ in $X$, respectively. Let $\mathbf{Z}(X)$ be the center of $X$. We need some properties regarding 2-transitive permutation groups, see [3].

Lemma 2.1 Let $H$ be a 2-transitive permutation group on $\Omega$. Assume that $N \triangleleft H$ is imprimitive on $\Omega$. Then $H$ is affine with $\operatorname{soc}(H)=\mathbb{Z}_{p}^{e}$, where $p$ is a prime and $e \geq 1$, and further, the following hold:

(i) An imprimitive normal subgroup of $H$ is of the form $\mathbb{Z}_{p}^{e} \cdot \mathbb{Z}_{b}$, where $b \mid p^{e^{\prime}}-1$ and $e^{\prime}$ is a proper divisor of $e$.

(ii) If $N$ is a subnormal subgroup of $H$, then either $N$ is transitive on $\Omega$, or $N<$ $\operatorname{soc}(H)$.

(iii) $H_{\omega}$ has no non-trivial normal subgroup of $p$-power order, for $\omega \in \Omega$. 
Let $\Gamma$ be an $(X, 2)$-arc-transitive graph with vertex set $V$. For $v \in V$, let $\Gamma(v)=$ $\{w \in V \mid(v, w)$ is an arc $\}$, the neighborhood of $v$. As usual, let $X_{v}^{[1]}$ be the kernel of $X_{v}$ acting on $\Gamma(v)$, and let $X_{v}^{\Gamma(v)}$ be the permutation group induced by $X_{v}$ on $\Gamma(v)$. Then $X_{v}^{\Gamma(v)} \cong X_{v} / X_{v}^{[1]}$. For $w \in \Gamma(v)$, let $X_{v w}=X_{v} \cap X_{w}$, and $X_{v w}^{[1]}=X_{v}^{[1]} \cap X_{w}^{[1]}$, the kernel of $X_{v w}$ acting on $\Gamma(v) \cup \Gamma(w)$. We observe that $X_{v} \cong X_{v}^{[1]} \cdot X_{v}^{\Gamma(v)}$, and $\left(X_{v}^{[1]}\right)^{\Gamma(w)} \cong\left(X_{v}^{[1]} X_{w}^{[1]}\right) / X_{w}^{[1]} \cong X_{v}^{[1]} / X_{v w}^{[1]}$. Thus $X_{v}^{[1]} \cong X_{v w}^{[1]} \cdot\left(X_{v}^{[1]}\right)^{\Gamma(w)}$, and so

$$
X_{v} \cong X_{v}^{[1]} \cdot X_{v}^{\Gamma(v)} \cong\left(X_{v w}^{[1]} \cdot\left(X_{v}^{[1]}\right)^{\Gamma(w)}\right) \cdot X_{v}^{\Gamma(v)} .
$$

In particular, if $X_{v w}^{[1]}=1$, then

$$
\left\{\begin{array}{l}
X_{v}^{[1]} \cong\left(X_{v}^{[1]}\right)^{\Gamma(w)}, \\
X_{v} \cong\left(X_{v}^{[1]}\right)^{\Gamma(w)} \cdot X_{v}^{\Gamma(v)} .
\end{array}\right.
$$

Here we have a fundamental theorem for studying 2-arc transitive graphs, which can be found in [17].

Theorem 2.2 Let $\Gamma$ be an $(X, 2)$-arc transitive graph, and let $\{v, w\}$ be an edge of $\Gamma$. Then either $X_{v w}^{[1]}=1$, or $X_{v w}^{[1]}$ is a $p$-group with $p$ prime and $\operatorname{PSL}(d, q) \triangleleft X_{v}^{\Gamma(v)}$ with $|\Gamma(v)|=\frac{q^{d}-1}{q-1}$.

\section{Proof of the main theorem}

Let $\Gamma$ be an $(X, 2)$-arc transitive graph with vertex set $V$. Assume that $X$ is quasiprimitive on $V$ of type TW. Let $G=\operatorname{soc}(X)$, the unique minimal normal subgroup of $X$, which is regular on $V$. Let $n=|V|=|G|$.

We first treat the case where the centralizer $\mathbf{C}_{\mathrm{Aut} \Gamma}(G)$ is non-trivial.

Lemma 3.1 Assume that $\mathbf{C}_{\mathrm{Aut} \Gamma}(G) \neq 1$. Then $\Gamma$ has valency 8 , and Aut $\Gamma=(G \times$ $\left.\mathbb{Z}_{2}^{3}\right) \rtimes \operatorname{GL}(3,2)=G \rtimes \operatorname{AGL}(3,2)$.

Proof Let $C=\mathbf{C}_{\text {Aut } \Gamma}(G), E=C G$, and $F=C X$. Since $G$ is regular, we have that $E=C \times G$, and $G, E \triangleleft F$. Thus $X \cap E=G$, and so $X_{v} \cap E_{v}=1$ and $F_{v}=E_{v} \rtimes X_{v}$. Since $\Gamma$ is connected, $F_{v}$ acts faithfully on $\Gamma(v)$, and hence $X_{v} \cong X_{v}^{\Gamma(v)} \leq F_{v}^{\Gamma(v)} \cong$ $F_{v}$, and $X_{v}$ and $F_{v}$ are both 2-transitive permutation groups on $\Gamma(v)$. Inspecting the 2transitive permutation groups, see [3], it is easily concluded that $E_{v} \cong \mathbb{Z}_{2}^{3}$, and $X_{v} \cong$ $\operatorname{GL}(3,2) \cong \operatorname{PSL}(2,7)$, which is of degree 8. Thus $F_{v}=\mathbb{Z}_{2}^{3} \rtimes \operatorname{GL}(3,2)=\operatorname{AGL}(3,2)$, and $\Gamma$ has valency 8 . Finally, $C \cong E / G \cong E_{v} \cong \mathbb{Z}_{2}^{3}$, and $C \rtimes X_{v} \cong((C \times G) \rtimes$ $\left.X_{v}\right) / G=F / G \cong F_{v}=\operatorname{AGL}(3,2)$. Since $\operatorname{AGL}(3,2)$ acts irreducibly on its natural module $\mathbb{Z}_{2}^{3}$, it follows that $C$ is a minimal normal subgroup of $F$.

Let $Y=\mathbf{N}_{\text {Aut } \Gamma}(G)$. Then $F_{v} \leq Y_{v}$, and $Y_{v}$ is a 2-transitive permutation group on $\Gamma(v)$. Suppose that $F_{v}<Y_{v}$. It follows since $F_{v}=\operatorname{AGL}(3,2)$ that $Y_{v}=\mathrm{A}_{8}$ or $\mathrm{S}_{8}$. However, since $G \triangleleft Y$ and $G$ is non-Abelian and characteristically simple, $C=\mathbf{C}_{Y}(G) \cong C G / G \triangleleft Y / G \cong Y_{v}$, which is a contradiction. Therefore, $F_{v}=Y_{v}$ and so $F=Y=\mathbf{N}_{\text {Aut } \Gamma}(G)$. 
Suppose that $F<\operatorname{Aut} \Gamma$. Let $Z$ be a group such that $F<Z \leq \operatorname{Aut} \Gamma$, and $F$ is a maximal subgroup of $Z$. Then $\operatorname{AGL}(3,2)=F_{v}^{\Gamma(v)} \leq Z_{v}^{\Gamma(v)} \leq \operatorname{Sym}(\Gamma(v)) \cong \mathrm{S}_{8}$. It follows that $Z_{v}^{\Gamma(v)}=\operatorname{AGL}(3,2), \mathrm{A}_{8}$ or $\mathrm{S}_{8}$, and $Z_{v w}^{\Gamma(w)} \cong \mathrm{GL}(3,2), \mathrm{A}_{7}$ or $\mathrm{S}_{7}$, respectively. Since $\left(Z_{v}^{[1]}\right)^{\Gamma(w)} \unlhd Z_{v w}^{\Gamma(w)}$, we have that $\left(Z_{v}^{[1]}\right)^{\Gamma(w)}=1, \operatorname{GL}(3,2), \mathrm{A}_{7}$ or $\mathrm{S}_{7}$. By Theorem 2.2, we conclude that $Z_{v w}^{[1]}=1$, and hence $Z_{v} \cong\left(Z_{v}^{[1]}\right)^{\Gamma(w)} \cdot Z_{v}^{\Gamma(v)}$. It then follows that either $Z_{v} \cong Z_{v}^{\Gamma(v)}$, or $Z_{v} \cong Z_{v w}^{\Gamma(w)} \times Z_{v}^{\Gamma(v)}$. Since $Z_{v}>F_{v}$ and $F_{v} \cong \operatorname{AGL}(3,2), \mathrm{A}_{8}$ or $\mathrm{S}_{8}$, it is easily shown that $Z_{v}$ is one of the following:

$$
A_{8}, \quad S_{8}, \quad \operatorname{GL}(3,2) \times \operatorname{AGL}(3,2), \quad A_{7} \times A_{8}, \quad A_{7} \times S_{8}, \quad S_{7} \times S_{8} .
$$

As $F$ and $Z$ are transitive on $V, \frac{|Z|}{|F|}=\frac{\left|Z_{v}\right|}{\left|F_{v}\right|}$ is one of the following numbers:

$$
2^{3} 3.7, \quad 2^{3} 3^{2} 5.7, \quad 2^{4} 3^{2} 5.7, \quad 3.5, \quad 2.3 .5, \quad 2^{3} 3^{3} 5^{2} 7, \quad 2^{4} 3^{3} 5^{2} 7 \quad \text { or } \quad 2^{5} 3^{3} 5^{2} 7 \text {. }
$$

Since $G$ is a characteristic subgroup of $F$ and $G$ is not normal in $Z$, it follows that $F$ is not normal in $Z$. Let $\Omega=[Z: F]$, the set of right cosets of $F$ in $Z$. Since $F$ is a maximal subgroup of $Z$, the action of $Z$ on $\Omega$ is primitive. As $G$ is not normal in $Z$, and as $G, C$ are the only minimal normal subgroups of $F$, it follows that the kernel of this $Z$-action on $\Omega$ is trivial or equals $C \cong \mathbb{Z}_{2}^{3}$. If the kernel equals $C$, then it follows that $Z$ centralizes $C$. However, $X$ does not centralizes $C$ and $Z>X$, which is a contradiction. Therefore, $Z$ is a primitive permutation group on $\Omega$. Then since $|\Omega|=|Z: F|$ is not a proper power of an integer, it follows from the O'Nan-Scott theorem that $Z$ is an almost simple group. Since $Z=G Z_{v}$, by [2, Theorem 1.4], $\operatorname{soc}(Z) \cong \mathrm{A}_{n}$ where $n=|G|$, and so $\Gamma$ is a complete graph, which is a contradiction.

Therefore, Aut $\Gamma=F=G \rtimes \operatorname{AGL}(3,2)$.

From now on, we assume that

$$
\mathbf{C}_{\text {Aut } \Gamma}(G)=1 \text {. }
$$

Suppose that $G$ is not normal in Aut $\Gamma$. Then $X \neq \mathrm{Aut} \Gamma$, and there exists $Z \leq \mathrm{Aut} \Gamma$ such that $X$ is a maximal subgroup of $Z$. Let $N$ be a minimal normal subgroup of $Z$. Then $N \not \subset X$. Write $N=T_{1} \times \ldots \times T_{l}$, where $l \geq 1$ and $T_{1} \cong \ldots \cong T_{l}$ are simple groups. We use a series of steps to derive a contradiction.

Step 1. $N$ is non-Abelian.

Suppose that $N$ is Abelian, say $N \cong \mathbb{Z}_{p}^{l}$ with $p$ prime. Let $E=N G=N \rtimes G$, and let $F=N X=N \rtimes X$. Since $G$ is regular on $V$, we have that $\left|E_{v}\right|=|N|=p^{l}$, and $E_{v}$ is a $p$-group. It follows that $E_{v}^{\Gamma(v)}$ is a non-trivial $p$-group. Since $E \triangleleft F$, we have $1 \neq E_{v}^{\Gamma(v)} \triangleleft F_{v}^{\Gamma(v)}$, and since $F_{v}^{\Gamma(v)}$ is a 2-transitive permutation group, $F_{v}^{\Gamma(v)}$ is affine. It then follows that $E_{v} \cong E_{v}^{\Gamma(v)}$, and $\operatorname{soc}\left(F_{v}^{\Gamma(v)}\right)=E_{v}^{\Gamma(v)} \cong \mathbb{Z}_{p}^{l}$. By Theorem 2.2, $F_{v w}^{[1]}=1$, and hence by Formula (2),

$$
p^{l}=|N|=\frac{|F|}{|X|}=\frac{\left|F_{v}\right|}{\left|X_{v}\right|}=\frac{\left|F_{v}^{\Gamma(v)}\right|}{\left|X_{v}^{\Gamma(v)}\right|} \cdot\left|\left(F_{v}^{[1]}\right)^{\Gamma(w)}\right| .
$$


Now $X_{v}^{\Gamma(v)}$ and $F_{v}^{\Gamma(v)}$ are affine 2-transitive permutation groups of degree $p^{l}$ such that $X_{v}^{\Gamma(v)}$ is a subgroup of $F_{v}^{\Gamma(v)}$ of index dividing $p^{l}$, and $\left(F_{v}^{[1]}\right)^{\Gamma(w)}$ is a normal subgroup of $F_{v w}^{\Gamma(w)}$ of order dividing $p^{l}$. Inspecting the classification of 2-transitive permutation groups of degree $p^{l}$ (see [3]), we conclude that $F_{v}^{\Gamma(v)}=X_{v}^{\Gamma(v)}$ and $\left(F_{v}^{[1]}\right)^{\Gamma(v)}=1$, which is a contradiction. Thus $N$ is non-Abelian.

Step 2. $N$ is transitive on $V$, and either $G<N$, or each prime divisor $r$ of $|T|$ is such that $r^{l} \quad \chi|V|$.

If $G<N$, then $N$ is transitive on $V$. Suppose that $G \nless N$. Since $G$ is a minimal normal subgroup of $X$ and $G \cap N$ is normalized by $X$, it follows that $G \cap N=$ 1. Since $\mathbf{C}_{\mathrm{Aut} \Gamma}(G)=1$ and $G$ is insoluble, $G$ and so $X$ permutes by conjugation $\left\{T_{1}, \ldots, T_{l}\right\}$ non-trivially. As $G$ is a minimal normal subgroup of $X$, it follows that $G$ permutes by conjugation the collection $\left\{T_{1}, \ldots, T_{l}\right\}$, and so $G \leq \operatorname{Sym}(l)$. In particular, $|G|$ divides $l$ !.

Suppose that there exists a prime $r$ dividing $|T|$ such that $r^{l}$ divides $|V|$. Then $r^{l}$ divides $|G|$, and so $r^{l}$ divides $l$ !. However, it is known that the highest power of $r$ dividing $l$ ! is at most $r^{\frac{l-1}{r-1}}$. Hence $r^{l} \leq r^{\frac{l-1}{r-1}}$, and so $l \leq \frac{l-1}{r-1} \leq l-1$, which is not possible. Thus, if a prime $r|| T \mid$, then $r^{l} \quad \chi|V|$. In particular, $N$ is not semiregular on $V$. Since $\Gamma$ is not bipartite, it follows that $N$ is transitive on $V$.

Step 3. $Z$ is quasiprimitive on $V$ of type $P A$, and $G<N$.

By Step 2, any minimal normal subgroup of $Z$ is transitive on $V$, and so $Z$ is quasiprimitive on $V$, which has an irregular minimal normal subgroup $N$. By [13], $Z$ is of type HA, AS, TW or PA. Since $N$ is not regular, $Z$ is neither of type HA nor of type TW. If $Z=G Z_{v}$ is almost simple, then by [2, Theorem 1.4], $\operatorname{soc}(Z) \geq \mathrm{A}_{n}$ where $n=|V|$ and $Z$ is 2-transitive on $V$. Thus $\Gamma$ is a complete graph, and so $X$ is 2-transitive on $V$, which is not possible. Thus $Z$ is of type PA, and so there exists a prime $r|| T \mid$ such that $r^{l}|| V \mid$. By Step 2, $G<N$.

Step 4. The socle $\operatorname{soc}\left(X_{v}\right) \leq N_{v}$.

Suppose $\operatorname{soc}\left(X_{v}\right) \not N_{v}$. Let $Y=N X$. Then $N \cap X_{v}=1$, and $N \triangleleft Y$. Hence $N_{v} \triangleleft Y_{v}$, and $Y_{v} / N_{v} \cong Y / N \cong X_{v}$. So $Y_{v}=N_{v} \rtimes X_{v}$; in particular, $|\Gamma(v)|^{2}$ divides $\left|Y_{v}\right|$. Suppose that $Y_{v w}^{[1]}=1$. Then by Formula (2), $Y_{v} \cong\left(Y_{v}^{[1]}\right)^{\Gamma(w)} \cdot Y_{v}^{\Gamma(v)}$. Noting that $\left(Y_{v}^{[1]}\right)^{\Gamma(w)} \triangleleft G_{v w}^{\Gamma(w)}$, it is easily shown that $Y_{v}$ does not have the form $N_{v} \rtimes X_{v}$ such that both $N_{v}$ and $X_{v}$ are transitive on $\Gamma(v)$, which is not possible. Thus $Y_{v w}^{[1]} \neq 1$, and by Theorem 2.2, $\operatorname{soc}\left(Y_{v}^{\Gamma(v)}\right)=\operatorname{PSL}(d, q)$ with $|\Gamma(v)|=\frac{q^{d}-1}{q-1}$. Since $Y_{v w}^{[1]}$ is a $p$-group and $\left(Y_{v}^{[1]}\right)^{\Gamma(w)} \triangleleft G_{v w}^{\Gamma(w)}$, it follows that $|\Gamma(v)|^{2}$ does not divide $\left|Y_{v}\right|$, which is a contradiction. Thus, $\operatorname{soc}\left(X_{v}\right) \leq N_{v}$.

Let $G=S_{1} \times \ldots \times S_{k}$, where $k \geq 2$ and $S_{1} \cong \ldots \cong S_{k}$ are non-Abelian simple groups. Then $X_{v}$ acts by conjugation transitively on $\left\{S_{1}, \ldots, S_{k}\right\}$, and $\operatorname{soc}\left(X_{v}\right)$ is half-transitive on $\left\{S_{1}, \ldots, S_{k}\right\}$, that is, all orbits of $\operatorname{soc}\left(X_{v}\right)$ on $\left\{S_{1}, \ldots, S_{k}\right\}$ have equal size. 
Step 5. $k=l$, and for each $i \in\{1,2, \ldots, l\}, T_{i} \cap G=S_{j}$ for some $1 \leq j \leq k$.

Let $H=T_{1} \times \ldots \times T_{k-1}$. Since $Z$ is of type PA, the proper normal subgroup $H$ is intransitive on $V$. Let $\mathcal{B}$ be the set of $H$-orbits on $V$, and let $B \in \mathcal{B}$ contain $v$. Then $N^{\mathcal{B}} \cong N / H \cong T$, and $G^{\mathcal{B}} \cong G /(G \cap H) \cong S^{m}$ where $m \geq 1$. In particular, $|S|$ divides $|T|$. For convenience, write $\bar{T}=N^{\mathcal{B}} \cong T$. Now $G^{\mathcal{B}}$ is a transitive subgroup of $N^{\mathcal{B}}$, so $T \cong \bar{T}=G^{\mathcal{B}} \bar{T}_{B} \cong S^{m} \bar{T}_{B}$.

Suppose that $m>1$. By [2, Theorem 1.4], $\bar{T} \cong \mathrm{A}_{n}$ and $\bar{T}_{B} \cong \mathrm{A}_{n-1}$ with $n \geq 10$. Let $r$ be the largest prime which is less than $n$. Then $r$ exactly divides $|\bar{T}|$ and $\left|\bar{T}_{B}\right|$. So $r$ does not divide $|S|$. Further, $r^{l}$ exactly divides $|N|$, and so $r^{l}$ divides $\left|N_{v}\right|$. If $N_{v}$ is primitive on $\Gamma(v)$, then $H$ is semiregular on $V$, and $N_{v} \cong \bar{T}_{B}$, which is a contradiction. Hence $N_{v}$ is imprimitive on $\Gamma(v)$. By Lemma 2.1, $N_{v}$ is soluble, and so $\bar{T}_{B} \cong N_{v} / H_{v}$ is soluble, which is again a contradiction.

Thus $m=1$, and so $S^{k-1}<T^{l-1}$. Since $G \cap H \triangleleft G$, we may assume that $S_{1} \times$ $\ldots \times S_{k-1}<H$. If $S_{k} \nless T_{l}$, then as $T_{l} \cap G \triangleleft G$ we have $T_{l} \cap G=1$. Thus $S^{k}=G<$ $N / T_{k} \cong T^{l-1}$, which is not possible. Hence $S_{k}<T_{l}$, and $T_{l} \cap G=S_{k}$. It follows that for each $i, T_{i} \cap G=S_{j}$ for some $j$, and $k=l$.

Step 6. It is not possible for $G$ not to be normal in Aut $\Gamma$.

It follows from Step 5 that $\left|N_{v}\right|=|T: S|^{k}$. Since $\operatorname{soc}\left(X_{v}\right) \leq N_{v}$, it follows that $\operatorname{soc}\left(X_{v}\right)$ acts trivially on $\left\{S_{1}, \ldots, S_{k}\right\}$, and so $\operatorname{soc}\left(X_{v}\right)$ normalizes each $S_{j}$. Since $\mathbf{C}_{X}(G)=1$, we conclude that $\operatorname{soc}\left(X_{v}\right) \leq \operatorname{Out}\left(S_{j}\right)$. In particular, $\operatorname{soc}\left(X_{v}\right)$ is soluble, by 'Schreier's Conjecture', and so $X_{v}$ is an affine 2-transitive permutation group on $\Gamma(v)$, say degree $|\Gamma(v)|=p^{e}$ with $p$ prime. Inspecting outer-automorphism groups of simple groups, we conclude that either $p^{e}=2^{3}$, or $e \leq 2$. It follows that $N_{v}^{\Gamma(v)} \geq$ $\operatorname{soc}\left(Y_{v}^{\Gamma(v)}\right)=\mathbb{Z}_{p}^{e}, \mathrm{~A}_{p^{e}}$, or $\operatorname{PSL}(d, q)$ with either $e=1$ and $\frac{q^{d}-1}{q-1}=p$ or $p^{e}=2^{3}$. For the former two cases, $N_{v w}^{[1]} \triangleleft Y_{v w}^{[1]}=1$, and hence $N_{v} \cong\left(N_{v}^{[1]}\right)^{\Gamma(w)} \cdot N_{v}^{\Gamma(v)}$. It is now easily shown that $\left|N_{v}\right|$ is not a proper power of an integer, which is a contradiction. Thus $N_{v}^{\Gamma(v)} \geq \operatorname{soc}\left(Y_{v}^{\Gamma(v)}\right)=\operatorname{PSL}(d, q)$ or $\operatorname{PSL}(2,7)$; in particular, $\Gamma$ is $(N, 2)$-arc transitive. If $\operatorname{soc}\left(Y_{v}^{\Gamma(v)}\right)=\operatorname{PSL}(d, q)$, then $p=\frac{q^{d}-1}{q-1}$ divides $\left|N_{v}\right|$, but $p^{2}$ does not, so $\left|N_{v}\right|$ is not a proper power of an integer, which is a contradiction. Thus $\operatorname{soc}\left(Y_{v}^{\Gamma(v)}\right)=$ $\operatorname{PSL}(2,7)$. It follows that $\left|N_{v}\right|$ is not a proper power of an integer, which is again a contradiction.

Now we summarize the argument for proving Theorem 1.1 as follows.

Proof of Theorem 1.1: By Steps 1-6, we have that $G$ is normal in Aut $\Gamma$. If $\mathbf{C}_{\text {Aut } \Gamma}(G) \neq$ 1, then by Lemma 3.1, part (i) of Theorem 1.1 holds. If $\mathbf{C}_{\mathrm{Aut} \Gamma}(G)=1$, then $G$ is the unique minimal normal subgroup of $A u t \Gamma$, as in part (ii) of Theorem 1.1. Finally, by [9, Proposition 2.3], $\Gamma$ is not 3-arc transitive.

\section{Some related problems}

Let $\Gamma$ be an $X$-arc transitive graph with vertex set $V \Gamma$. Let $N \triangleleft X$ have at least three orbits on $V \Gamma$, and let $\mathcal{B}$ be the set of $N$-orbits in $V \Gamma$. We define a graph $\Gamma_{N}$ to have 
vertex set $\mathcal{B}$ such that $B, C \in \mathcal{B}$ are adjacent if and only if some $u \in B$ is adjacent in $\Gamma$ to some $v \in C$, called the normal quotient of $\Gamma$ induced by $N$. If $\Gamma$ and $\Gamma_{N}$ have equal valency, then $\Gamma$ is a normal cover of $\Gamma_{N}$. An $(X, 2)$-arc transitive graph $\Gamma$ is always a normal cover of $\Gamma_{N}$, and choosing $N$ to be maximal, each non-trivial normal subgroup of $X / N$ is transitive on $V \Gamma_{N}$, that is, $X / N$ is quasiprimitive on $V \Gamma_{N}$. This is the reduction for the study of 2-arc transitive graphs to the quasiprimitive case, given by Praeger [13].

A graph $\Gamma$ with vertex set $V$ is called a Cayley graph if there exist a group $G$ and a subset $S \subset G$ with $S=S^{-1}=\left\{s^{-1} \mid s \in S\right\}$ such that $V$ is identified with $G$ and $x, y \in G$ are adjacent if and only if $y x^{-1} \in S$. The Cayley graph $\Gamma$ is denoted by $\operatorname{Cay}(G, S)$.

A Cayley graph $\Gamma=\operatorname{Cay}(G, S)$ has an automorphism group

$$
\hat{G}=\{\hat{g}: x \mapsto x g \text { for all } x \in G \mid g \in G\},
$$

consisting of right multiplications of elements of $G$. The subgroup $\hat{G}$ acts regularly on the vertex set of $\Gamma$; in particular, $\Gamma$ is vertex-transitive. Let

$$
\operatorname{Aut}(G, S)=\left\{\sigma \in \operatorname{Aut}(G) \mid S^{\sigma}=S\right\}
$$

the subgroup of automorphisms of $G$ and fix $S$ setwise. It is easily shown that all elements of Aut $(G, S)$ are automorphisms of $\Gamma$ fixing the vertex of $\Gamma$ corresponding to the identity of $G$. If $\Gamma$ is connected, or equivalently $\langle S\rangle=G$, then $\operatorname{Aut}(G, S)$ acts faithfully on $S$. Further, $\operatorname{Aut}(G, S)$ normalizes $\hat{G}$, and

$$
\mathbf{N}_{\text {Aut } \Gamma}(\hat{G})=\hat{G} \rtimes \operatorname{Aut}(G, S) \leq \operatorname{Aut} \Gamma .
$$

Thus, if $\operatorname{Aut}(G, S)$ is 2-transitive on $S$ then $\Gamma$ is $\left(\mathbf{N}_{\text {Aut } \Gamma}(\hat{G}), 2\right)$-arc-transitive.

Much structural information of $\Gamma$ is contained in the full automorphism group Aut $\Gamma$, such as the degree of symmetry of $\Gamma$, and the isomorphism class of $\Gamma$ among Cayley graphs of $G$ (refer to [10]). Generally, Aut $\Gamma$ is larger than $\hat{G} \rtimes \operatorname{Aut}(G, S)$, see for example $[4,6,11,16]$. Theorem 1.1 can be restated in the Cayley graph version.

Theorem 4.1 Let $G$ be a non-Abelian characteristically simple group, and let $\Gamma=$ Cay $(G, S)$ be a connected Cayley graph. Assume that there exists a subgroup $X \leq$ Aut $\Gamma$ such that $\hat{G}$ is a unique minimal normal subgroup of $X$, and assume further that $\Gamma$ is $(X, 2)$-arc-transitive. Then $\operatorname{Aut} \Gamma=\hat{G} \rtimes \operatorname{Aut}(G, S), \Gamma$ is not 3-arc transitive, and further either

(i) $\Gamma$ has valency 8 , and Aut $\Gamma=\left(\hat{G} \times \mathbb{Z}_{2}^{3}\right) \rtimes \operatorname{GL}(3,2)$; or

(ii) $\hat{G}$ is the unique minimal normal subgroup of Aut $\Gamma$.

A natural question is whether the conclusion of Theorem 4.1 is always true for arbitrary groups $G$. Here is a counter-example.

Example 4.2 Let $G=N \rtimes\langle z\rangle \cong \mathbb{Z}_{p}^{e} \rtimes \mathbb{Z}_{2}$ with $p$ odd prime and $e \geq 1$ such that $x^{z}=x^{-1}$ for all $x \in N$. Let $S=G \backslash N$, and let $\Gamma=\operatorname{Cay}(G, S)$. Then $S$ consists of all involutions of $G$ which are conjugate, and $\Gamma=\mathbf{K}_{p^{e}}, p^{e}$, a complete bipartite 
graph. It is easily shown that $\operatorname{Aut}(G, S)=\operatorname{Aut}(G) \cong \operatorname{AGL}(e, p)=\mathbb{Z}_{p}^{e} \rtimes \operatorname{GL}(e, p)$ and $\operatorname{Aut}(G, S)$ acts 2-transitively on $S$. However, Aut $\Gamma \cong \mathrm{S}_{p^{e}}>\mathrm{S}_{2}$ is much bigger than $\mathbf{N}_{\text {Aut } \Gamma}(\hat{G})=\hat{G} \rtimes \operatorname{AGL}(e, p)$.

However, $\mathbf{K}_{p^{e}, p^{e}}$ and their normal covers are the only known examples such that $\operatorname{Aut}(G, S)$ is 2-transitive and $\hat{G}$ is not normal in Aut $\Gamma$. This motivates the following conjecture:

Conjecture 4.3 For a connected Cayley graph $\Gamma=\operatorname{Cay}(G, S)$, if $\operatorname{Aut}(G, S)$ is 2-transitive on $S$, then either $\Gamma$ is a normal cover of $\mathbf{K}_{p^{e}}, p^{e}$ with $p$ odd prime, or $\operatorname{Aut} \Gamma=\hat{G} \rtimes \operatorname{Aut}(G, S)$.

For a group $G$, a Cayley graph $\Gamma=\operatorname{Cay}(G, S)$ is called normal if $\hat{G}$ is normal in Aut $\Gamma$, see [18]. Then Conjecture 4.3 means that most Cayley graphs $\operatorname{Cay}(G, S)$ are normal provided that $\operatorname{Aut}(G, S)$ is 2-transitive on $S$.

More generally, we would like to propose several further problems regarding automorphism groups of Cayley graphs. We believe their solutions would be interesting for a better understanding of Cayley graphs.

Naturally, one would ask whether the condition in Conjecture 4.3 that $\operatorname{Aut}(G, S)$ is 2-transitive on $S$ can be weakened to the condition that $\operatorname{Aut}(G, S)$ is only transitive on $S$, refer to $[4,11,16,18]$.

Problem 4.4 Characterize connected Cayley graphs $\Gamma=\operatorname{Cay}(G, S)$ such that Aut $(G, S)$ is transitive on $S$ and $\operatorname{Aut} \Gamma \neq \hat{G} \rtimes \operatorname{Aut}(G, S)$. Especially, do this for the case $\operatorname{Aut}(G, S)$ is primitive on $S$.

By [16, Theorem 1], Problem 4.4 was solved for the case where $G$ is non-Abelian simple and $\Gamma$ is cubic, that is, it was shown that if $G$ is a non-Abelian simple group and $\operatorname{Aut}(G, S)$ is transitive on $S$ with $|S|=3$ then $\operatorname{Aut} \Gamma=\hat{G} \rtimes \operatorname{Aut}(G, S)$.

Example 4.5 For a Fermat prime $p=2^{d}-1$, the complete graph $\Gamma=\mathbf{K}_{2^{d}}$ of $2^{d}$ vertices is a Cayley graph $\operatorname{Cay}(G, S)$ of $G=\mathbb{Z}_{2}^{d}$ such that $\operatorname{Aut}(G, S) \cong \operatorname{GL}(d, 2)$, which is primitive on $S$, and Aut $\Gamma=\operatorname{Sym}\left(2^{d}\right)$. In particular, for $d \geq 3, \hat{G}$ is not normal in Aut $\Gamma$.

Cayley graphs $\operatorname{Cay}(G, S)$ with the property that $\operatorname{Aut}(G, S)$ is transitive on $S$ are called normal arc-transitive Cayley graphs. A study of such Cayley graphs was initiated in [16]. We wonder for a 2-arc-transitive Cayley graph Cay $(G, S)$ whether the transitivity of Aut $(G, S)$ on $S$ implies the 2-transitivity:

Question 4.6 Does there exist a 2-arc-transitive Cayley graph Cay $(G, S)$ such that Aut $(G, S)$ is transitive but not 2-transitive on $S$ ?

Cayley graphs defined above are undirected. Of course one may define directed Cayley graphs and ask similar questions regarding their automorphism groups to the undirected case. We are inclined to conjecture for a directed Cayley graph 
$\Gamma=\operatorname{Cay}(G, S)$, if $\operatorname{Aut}(G, S)$ is 2-transitive on $S$, then $\operatorname{Aut}(G, S)$ is also very close to $(A u t \Gamma)_{v}$ where $v$ is the vertex of $\Gamma$ corresponding to the identity of the group $G$.

Question 4.7 Let $\Gamma=\operatorname{Cay}(G, S)$ be a connected directed Cayley graph, and assume further that $\operatorname{Aut}(G, S)$ is 2 -transitive on $S$. Does Aut $\Gamma$ equal $\hat{G} \rtimes \operatorname{Aut}(G, S)$ ?

Praeger [14] gave a description of the bipartite 2-arc-transitive graphs. Let $\Gamma$ be an $(X, 2)$-arc-transitive bipartite graph with parts $\Delta$ and $\Delta^{\prime}$, where $X \leq \operatorname{Aut} \Gamma$. Let $X^{+}=X_{\Delta}=X_{\Delta^{\prime}}$. The graph $\Gamma$ is said to be $X$-bi-quasiprimitive if each non-trivial normal subgroup has at most two orbits and at least one has two orbits. It is shown in [14] that the bi-quasiprimitive case is an important case for understanding bipartite 2-arc-transitive graphs, and if $X^{+}$is bi-quasiprimitive then $X^{+}$is of type HA, AS, TW or PA. Motivated by Theorem 4.1, we propose

Conjecture 4.8 Let $\Gamma$ be a connected $(X, 2)$-arc-transitive bipartite graph with parts $\Delta$ and $\Delta^{\prime}$, where $X \leq$ Aut $\Gamma$. Assume further that $X^{+}$is quasiprimitive on $\Delta$ of type HA or type TW. Then either $\Gamma=\mathbf{K}_{p^{e}}, p^{e}$ with $p$ prime, or $\operatorname{soc}\left(X^{+}\right)$is normal in $(\text { Aut } \Gamma)^{+}$.

\section{References}

1. Baddeley, R.W.: Two-arc transitive graphs and twisted wreath products. J. Algebr. Comb. 2, 215-237 (1993)

2. Baddeley, R.W., Praeger, C.E.: On primitive overgroups of quasiprimitive permutation groups. J. Algebra 263, 291-361 (2003)

3. Cameron, P.: Permutation Groups. London Mathematical Society Student Texts, vol. 45, Cambridge University Press, Cambridge (1999)

4. Fang, X.G., Li, C.H., Wang, J., Xu, M.Y.: On cubic Cayley graphs of finite simple groups. Discrete Math. 244, 67-75 (2002)

5. Fang, X.G., Havas, G., Wang, J.: A family of non-quasiprimitive graphs admitting a quasiprimitive 2-arc transitive group action. Eur. J. Comb. 20, 551-557 (1999)

6. Godsil, C.D.: On the full automorphism group of a graph. Combinatorica 1, 243-256 (1981)

7. Ivanov, A.A., Praeger, C.E.: On finite affine 2-arc transitive graphs. Eur. J. Comb. 14, 421-444 (1993)

8. Li, C.H.: A family quasiprimitive 2-arc-transitive graphs which have non-quasiprimitive full automorphism groups. Eur. J. Comb. 19, 499-502 (1998)

9. Li, C.H.: Finite $s$-arc transitive graphs of prime-power order. Bull. Lond. Math. Soc. 33, 129-137 (2001)

10. Li, C.H.: On isomorphisms of finite Cayley graphs-a survey. Discrete Math. 256, 301-334 (2002)

11. Li, C.H.: On finite edge-transitive Cayley graphs and rotary Cayley maps. Trans. Am. Math. Soc. 358, 4605-4635 (2006)

12. Li, C.H., Seress, A.: Constructions of quasiprimitive two-arc transitive graphs of product action type. In: Finite Geometries, Groups and Computation, pp. 115-124 (2006)

13. Praeger, C.E.: An O'Nan-Scott theorem for finite quasiprimitive permutation groups and an application to 2-arc transitive graphs. J. Lond. Math. Soc. 47, 227-239 (1992)

14. Praeger, C.E.: On a reduction theorem for finite, bipartite 2 -arc-transitive graphs. Australas. J. Comb. 7, 21-36 (1993)

15. Praeger, C.E.: Finite quasiprimitive graphs. In: Surveys in Combinatorics 1997, London. London Mathematical Society Lecture Notes series, vol. 241, pp. 65-85. Cambridge University Press, Cambridge (1997) 
16. Praeger, C.E.: Finite normal edge-transitive Cayley graphs. Bull. Austral. Math. Soc. 60, 207-220 (1999)

17. Weiss, R.: $s$-transitive graphs. In: Algebraic methods in graph theory, vols. I, II, Szeged, 1978. Colloquia Mathematica Societatis Jnos Bolyai, vol. 25, pp. 827-847. North-Holland, Amsterdam (1981)

18. Xu, M.Y.: Automorphism groups and isomorphisms of Cayley digraphs. Discrete Math. 182, 309-320 (1998) 